\title{
Catheter-Directed Treatment for Pulmonary Embolism in Light of Current Evidence
}

\begin{abstract}
Pulmonary embolism (PE) is the leading cause of in-hospital morbidity and mortality and accounts for approximately 100,000 deaths in the United States and 300,000 deaths in Europe annually. Although societal guidelines for low- and high-risk PE are well established, the present management of submassive (intermediate)-risk PE is evolving. Catheter-directed thrombolysis (CDT) represents a viable treatment option for treatment of submassive PE given its ability to rapidly reduce right heart strain with an acceptably low rate of major hemorrhagic complication. The current review aims to discuss the existing guidelines and literature supporting CDT for PE and also to examine upcoming areas of future research to support its adoption in the algorithm for the management of submassive PE.
\end{abstract}

Keywords: CDT, pulmonary thrombolysis, submassive PE

\section{Introduction}

Pulmonary embolism (PE) is a life-threatening entity that occurs in approximately $60-70 / 10,000$ individuals. ${ }^{[1]}$ It is the leading cause of in-hospital morbidity and mortality and accounts for roughly 100,000 deaths in the United States per year. $^{[2,3]}$ In Europe, this figure is even larger, responsible for over 300,000 deaths annually, of which only $7 \%$ are diagnosed antemortem. ${ }^{[3]}$ Moreover, mortality related to PE may potentially be higher than currently estimated as the majority of sudden deaths are typically attributed to a cardiac origin as opposed to PE.

At present, the severity of PE is stratified into three risk thresholds: low, intermediate, (submassive), and high (massive). Low risk is defined as $\mathrm{PE}$ in the absence of right heart strain. Submassive is defined as PE resulting in right heart dysfunction without systemic arterial hypotension (systolic blood pressure [SBP] $>90 \mathrm{mmHg}$ ) and represents up to $25 \%$ of patients presenting with PE. ${ }^{[4]}$ Conversely, massive $\mathrm{PE}$ is defined by the presence of central $\mathrm{PE}$ associated with right ventricular (RV) strain and sustained systemic arterial

This is an open access journal, and articles are distributed under the terms of the Creative Commons AttributionNonCommercial-ShareAlike 4.0 License, which allows others to remix, tweak, and build upon the work non-commercially, as long as appropriate credit is given and the new creations are licensed under the identical terms.

For reprints contact: reprints@medknow.com hypotension (SBP $<90 \mathrm{mmHg}$ for at least $15 \mathrm{~min}$ ) and/or the presence of central PE requiring inotropic support. ${ }^{[5]}$

Risk stratification guides subsequent treatment and the expected outcome of a patient presenting with $\mathrm{PE}$. The goals of treatment are primarily focused on the reduction of mortality and secondarily on the prevention of $\mathrm{PE}$ recurrence and late-onset chronic thromboembolic pulmonary hypertension. ${ }^{[2]}$ The established guidelines for low-risk PE (i.e., anticoagulation) and high-risk PE (i.e., aggressive clot which includes systemic thrombolysis, catheter-directed thrombolysis (CDT), and/or surgical thrombectomy) are generally well-accepted strategies. ${ }^{[6]}$ In recent years, however, the primary area of uncertainty with respect to management has centered around submassive PE. Treatment algorithms ranging from anticoagulation alone to systemic thrombolysis have been studied for submassive PE with varied results and conclusions. ${ }^{[7-9]}$

CDT is an emerging treatment option that has recently shown promise for patients with submassive PE. ${ }^{[10]}$ CDT utilizes an endovascular approach to locally deliver a thrombolytic agent within the thrombus itself to facilitate fibrinolysis. This technique has shown to accelerate the reversal of right

How to cite this article: Ahmed O, Shadid A Catheter-directed treatment for pulmonary embolism in light of current evidence. Arab J Intervent Radiol 2018;2:46-9.

\section{Osman Ahmed, Alexandria Shadid ${ }^{1}$}

Department of Radiology, Section of Interventional Radiology, University of Chicago Medicine, ${ }^{1}$ Department of Radiology, Section of Interventional Radiology, Rush University Medical Centre, Chicago, IL, USA
Address for correspondence: Dr. Osman Ahmed, Department of Radiology, Section of Vascular and Interventional Radiology, The University of Chicago Medical Centre, 5841 S. Maryland Ave., MC 2026, Room Q-219, Chicago, IL 60637, USA. E-mail: osman1423@gmail.com

Access this article online

Website: www.arabjir.com

DOI: 10.4103/AJIR.AJIR_24_18 Quick Response Code:

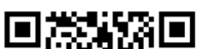

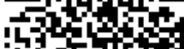
Sintory artaris? 
heart strain caused by $\mathrm{PE}$ at $24-48 \mathrm{~h}$ while also mitigating the bleeding risk associated with systemic thrombolysis. ${ }^{[3]}$ Direct delivery of a lytic agent into and around the clot can reduce the amount of drug being used, thereby reducing the risk of subsequent systemic bleeding. Nevertheless, the role of CDT in submassive PE continues to evolve, while prospective data remain limited. This review aims to examine the current CDT guidelines and existing literature for the treatment of PE and explore upcoming avenues of research in the field to potentially support its utilization.

In the past 3 years, three landmark prospective studies established the safety and efficacy of CDT for PE, laying the foundation for CDT in submassive PE: ULTIMA, SEATTLE II, and PERFECT..$^{[10-12]}$ ULTIMA was a prospective randomized trial that included 59 patients with central $\mathrm{PE}$ and echocardiographic RV-to-left ventricular (LV) diameter $(\mathrm{RV} / \mathrm{LV})$ ratio $\geq 1.0 .{ }^{[10]}$ Patients were randomized to receive unfractionated heparin only $(n=29)$ or systemic heparin along with ultrasound-assisted CDT (USAT) of $10-20 \mathrm{mg}$ of recombinant tissue-plasminogen activator (t-PA) infused over $15 \mathrm{~h}(n=30)$. The trial found USAT to be significantly superior to anticoagulation alone in reversing RV dilation at $24 \mathrm{~h}$, without an increase in bleeding complication. ${ }^{[10]}$ The significance of RV function is important as a meta-analysis by Cho et al. demonstrated that short-term mortality is increased for PE patients with RV dysfunction. ${ }^{[13]}$ SEATTLE II was a single-arm, multicenter trial that evaluated the safety and efficacy of USAT. The trial included 150 patients, 31 of which had acute massive PE and 119 had submassive proximal PE with a RV-to-LV diameter ratio on computed tomography $(\mathrm{CT}) \geq 0.9$. The trial similarly found that USAT decreased RV dilation, reduced pulmonary arterial hypertension, decreased anatomic thrombus burden, and minimized intracranial hemorrhage in patients with acute massive or submassive PE. ${ }^{[1]}$ PERFECT was a multicentric registry that prospectively enrolled 101 patients with acute PE ( $n=73$ submassive; $n=28$ massive) ${ }^{[12]}$ Of note, very few exclusion criteria were applied with the intention of presenting a real-world sampling of patients undergoing $\mathrm{CDT}^{\left[{ }^{[12]}\right.}$ Patients in the registry underwent either catheter-directed mechanical or pharmacomechanical thrombectomy and/or CDT with low-dose t-PA or urokinase. The registry similarly found that CDT resulted in improved hemodynamics with relief of right heart strain while reporting no major hemorrhagic or procedure-related complication.

Although these important studies have established the potential for CDT in the management of submassive PE, they remain limited in their ability to conclude definitively that CDT is a clinically beneficial treatment compared to alternative therapies. ${ }^{[14]}$ SEATTLE II and PERFECT were single-arm trials that lacked a comparator group to declare superiority. Both ULTIMA and SEATTLE II were fairly low sample size studies that were neither adequately designed nor powered to assess other clinically relevant variables as mortality or long-term impact on quality of life. Finally, PERFECT was limited by its nonrandomized design and also did not evaluate the long-term outcomes. ${ }^{[14]}$

Additional studies have aimed to identify the potential benefit of CDT in submassive PE. A recent meta-analysis by Mostafa et al. found that submassive patients undergoing CDT showed an all-cause mortality of $3.6 \%$ and risk of major bleeding of $0.9 \% .^{[15]}$ Additional small $(n=27-55)$ retrospective studies on submassive PE have found low rates of major hemorrhage $(0 \%-4 \%)$ and high rates of technical success. ${ }^{[16-18]}$

Given these limitations and the paucity of additional clinical data supporting CDT, the American College of Chest Physicians (ACCP) 2016 guidelines on venous thromboembolic disease recommend systemic thrombolysis over CDT unless patients are at higher risk for bleeding or are likely to deteriorate before systemic thrombolysis can take effect. These recommendations are however classified as Grade $2 \mathrm{C}$, signifying a weak recommendation based on a low level of evidence. ${ }^{[6]}$ Other societal guidelines from the American Heart Association and European Society of Cardiology recommend systemic thrombolysis or CDT only in patients in whom imminent cardiac decompensation is evident. ${ }^{[19]}$

Current societal recommendations such as the ACCP are in place despite established literature demonstrating a bleeding risk of up to $20 \%$ with systemic thrombolysis and intracranial hemorrhage risk of $3 \%-5 \%{ }^{[20]}$ Although CDT appears to mitigate the risk of major hemorrhage compared to systemic thrombolysis, the lack of clinical data supporting its safety in large numbers appears to have prohibited its adoption in contemporary guidelines. Given the need for further investigation, a research consensus panel was convened in December 2015 to determine what avenues of research are needed to justify the utilization of CDT for submassive PE. The panel determined that future trials should focus on identifying clinically impactful and feasible endpoints, determining appropriate selection criteria, maximizing enrollment, and collecting robust safety data regarding CDT. ${ }^{[21]}$

Choosing a feasible endpoint in the design of submassive $\mathrm{PE}$ trials remains challenging. Mortality would appear to represent a logical outcome measure; however, the reported rate of mortality in literature for submassive PE is varied. The International Cooperative Pulmonary Embolism Registry reported a mortality rate of up to $20 \%$; however, the more recent Pulmonary Embolism thrombolysis (PEITHO) trial demonstrated mortality in the range of $1.8 \%$. Furthermore, this $>1000$ patient trial along with two other meta-analyses did not demonstrate a mortality benefit when comparing systemic thrombolysis to anticoagulation alone. ${ }^{[8,9,22]}$ These results suggest that a large-scale trial to demonstrate mortality benefit with CDT may not be feasible. Alternatively, Sista 
et al. have suggested that dyspnea, exercise intolerance, and decreased quality of life following submassive PE may be a more reasonable outcome metric. ${ }^{[14]}$ These symptoms, collectively called "Post-PE syndrome," were shown to occur in a high percentage of patients following a first-time PE. ${ }^{[23,24]}$ Specifically, objective measures such as "6-min walk distance" may represent future endpoints to demonstrate the potential improvement in the quality of life when utilizing CDT for submassive PE. ${ }^{[14]}$

Triaging patients that may benefit from CDT also remains challenging. The present category of patients who were classified as "submassive" is heterogeneous and can range from asymptomatic individuals with an enlarged RV to acutely symptomatic patients with biochemical evidence of myocardial ischemia headed toward cardiac decompensation. Current prognostic models developed to assess PE mortality such as the Pulmonary Embolism Severity Index (PESI) were derived from low-risk patients and therefore may not be applicable to the submassive patient population and selecting those at risk for decompensation. ${ }^{[25]}$ Additional metrics such as RV/LV ratio and PA pressures are indirect measures that cannot reliably predict progression to $\mathrm{RV}$ failure. Recent literature has suggested that more specific models geared to assess the risk for cardiac arrest using vital signs, laboratory values, and age may be more accurate than PESI for predicting 30-day mortality. ${ }^{[25]}$ These scores may therefore be more beneficial in choosing patients that are more likely to benefit from CDT due to higher risk for mortality.

Finally, establishing robust safety data for CDT can help to offset its perceived invasive nature compared to systemic thrombolysis. Although systemic thrombolysis through a peripheral vein has shown to improve hemodynamics compared to anticoagulation alone, it must be weighed against the risk of major and intracranial hemorrhage. ${ }^{[26]}$ The PEITHO trial demonstrated a $11.5 \%$ incidence of bleeding in the systemic thrombolysis treatment arm, while a meta-analysis by Wang et al. demonstrated a $9.5 \%$ incidence of major hemorrhage. ${ }^{[27]}$ This elevated risk in addition to conflicting data surrounding mortality benefit is a likely contributing factor to why systemic lytic therapy is not recommended routinely in submassive PE. ${ }^{[6-9]}$ As continued research shows that CDT may offset the risk of bleeding, it can potentially be used to justify its utilization over systemic thrombolytics. To support this, a recent study by Avgerinos et al. retrospectively compared systemic thrombolysis to CDT for both submassive and massive $\mathrm{PE}$, finding improved safety and effect in the CDT arm. ${ }^{[2]}$ Another study by Kolkailah et al. demonstrated patients undergoing CDT to demonstrate a zero incidence of intracranial hemorrhage with slower Intensive Care Unit stays and hospital length of stay when compared with those undergoing pulmonary embolectomy. ${ }^{[26]}$ Similar studies performed prospectively will continue to support CDT's place over systemic thrombolysis for submassive PE.

\section{Conclusion}

The management of submassive PE is in a current state of evolution, with CDT representing a viable treatment option due to its low rate of hemorrhagic complication and ability to rapidly relieve right heart strain. However, additional prospective data are needed to further justify the use of CDT as a standard of care for submassive PE, specifically with respect to demonstrating superior clinically relevant outcome metrics compared to other alternative treatment options. Future studies will hopefully serve to cement the safety of CDT and also define better prognostic tests to identify patients in whom CDT will be most beneficial. Until then, CDT represents a viable option within the current guidelines for the management of submassive $\mathrm{PE}$ patients requiring escalation of care beyond systemic anticoagulation.

Financial support and sponsorship

Nil.

\section{Conflicts of interest}

There are no conflicts of interest.

\section{References}

1. Bĕlohlávek J, Dytrych V, Linhart A. Pulmonary embolism, part I: Epidemiology, risk factors and risk stratification, pathophysiology, clinical presentation, diagnosis and nonthrombotic pulmonary embolism. Exp Clin Cardiol 2013;18:129-38.

2. Avgerinos ED, Abou Ali AN, Liang NL, Rivera-Lebron B, Toma C, Maholic R, et al. Catheter-directed interventions compared with systemic thrombolysis achieve improved ventricular function recovery at a potentially lower complication rate for acute pulmonary embolism. J Vasc Surg Venous Lymphat Disord 2018;6:425-32.

3. Jolly M, Phillips J. Pulmonary embolism: Current role of catheter treatment options and operative thrombectomy. Surg Clin North Am 2018;98:279-92.

4. Kuo WT, Sista AK, Faintuch S, Dariushnia SR, Baerlocher MO, Lookstein RA, et al. Society of interventional radiology position statement on catheter-directed therapy for acute pulmonary embolism. J Vasc Interv Radiol 2018;29:293-7.

5. Jaff MR, McMurtry MS, Archer SL, Cushman M, Goldenberg N, Goldhaber SZ, et al. Management of massive and submassive pulmonary embolism, iliofemoral deep vein thrombosis, and chronic thromboembolic pulmonary hypertension: A scientific statement from the American heart association. Circulation 2011;123:1788-830.

6. Kearon C, Akl EA, Ornelas J, Blaivas A, Jimenez D, Bounameaux $\mathrm{H}$, et al. Antithrombotic therapy for VTE disease: CHEST guideline and expert panel report. Chest 2016;149:315-52.

7. Chatterjee S, Chakraborty A, Weinberg I, Kadakia M, Wilensky RL, Sardar P, et al. Thrombolysis for pulmonary embolism and risk of all-cause mortality, major bleeding, and intracranial hemorrhage: A meta-analysis. JAMA 2014;311:2414-21.

8. Meyer G, Vicaut E, Danays T, Agnelli G, Becattini C, Beyer-Westendorf $\mathrm{J}$, et al. Fibrinolysis for patients with intermediate-risk pulmonary embolism. N Engl J Med 2014;370:1402-11. 
9. Liu Y, Lu Y, Song J, Li D, Liu H, Yang J, et al. Recombinant tissue plasminogen activator for hemodynamically stable patients experiencing an acute pulmonary embolism: A meta-analysis. Thromb Res 2014;134:50-6.

10. Kucher N, Boekstegers P, Müller OJ, Kupatt C, Beyer-Westendorf J, Heitzer $\mathrm{T}$, et al. Randomized, controlled trial of ultrasound-assisted catheter-directed thrombolysis for acute intermediate-risk pulmonary embolism. Circulation 2014;129:479-86.

11. Piazza G, Hohlfelder B, Jaff MR, Ouriel K, Engelhardt TC, Sterling KM, et al. A prospective, single-arm, multicenter trial of ultrasound-facilitated, catheter-directed, low-dose fibrinolysis for acute massive and submassive pulmonary embolism: The SEATTLE II study. JACC Cardiovasc Interv 2015;8:1382-92.

12. Kuo WT, Banerjee A, Kim PS, DeMarco FJ Jr., Levy JR, Facchini FR, et al. Pulmonary embolism response to fragmentation, embolectomy, and catheter thrombolysis (PERFECT): Initial results from a prospective multicenter registry. Chest 2015;148:667-73.

13. Cho JH, Kutti Sridharan G, Kim SH, Kaw R, Abburi T, Irfan A, et al. Right ventricular dysfunction as an echocardiographic prognostic factor in hemodynamically stable patients with acute pulmonary embolism: A meta-analysis. BMC Cardiovasc Disord 2014;14:64.

14. Sista AK, Moriarty JM. The future of catheter-directed therapy: Data gaps, unmet needs, and future trials. Tech Vasc Interv Radiol 2017;20:224-6.

15. Mostafa A, Briasoulis A, Telila T, Belgrave K, Grines C. Treatment of massive or submassive acute pulmonary embolism with catheter-directed thrombolysis. Am J Cardiol 2016;117:1014-20.

16. Nassiri N, Jain A, McPhee D, Mina B, Rosen RJ, Giangola G, et al. Massive and submassive pulmonary embolism: Experience with an algorithm for catheter-directed mechanical thrombectomy. Ann Vasc Surg 2012;26:18-24.

17. Dumantepe M, Teymen B, Akturk U, Seren M. Efficacy of rotational thrombectomy on the mortality of patients with massive and submassive pulmonary embolism. J Card Surg 2015;30:324-32.
18. Fuller TJ, Paprzycki CM, Zubair MH, Hussain LR, Kuhn BA, Recht $\mathrm{MH}$, et al. Initial experiences with endovascular management of submassive pulmonary embolism: Is it safe? Ann Vasc Surg 2017;38:158-63.

19. Kesselman A, Kuo WT. Catheter-directed therapy for acute submassive pulmonary embolism: Summary of current evidence and protocols. Tech Vasc Interv Radiol 2017;20:193-6.

20. Kuo WT, Gould MK, Louie JD, Rosenberg JK, Sze DY, Hofmann LV, et al. Catheter-directed therapy for the treatment of massive pulmonary embolism: Systematic review and meta-analysis of modern techniques. J Vasc Interv Radiol 2009;20:1431-40.

21. Sista AK, Goldhaber SZ, Vedantham S, Kline JA, Kuo WT, Kahn SR, et al. Research priorities in submassive pulmonary embolism: Proceedings from a multidisciplinary research consensus panel. J Vasc Interv Radiol 2016;27:787-94.

22. Riera-Mestre A, Becattini C, Giustozzi M, Agnelli G. Thrombolysis in hemodynamically stable patients with acute pulmonary embolism: A meta-analysis. Thromb Res 2014;134:1265-71.

23. Kahn SR, Hirsch AM, Akaberi A, Hernandez P, Anderson DR, Wells PS, et al. Functional and exercise limitations after a first episode of pulmonary embolism: Results of the ELOPE prospective cohort study. Chest 2017;151:1058-68.

24. Sista AK, Miller LE, Kahn SR, Kline JA. Persistent right ventricular dysfunction, functional capacity limitation, exercise intolerance, and quality of life impairment following pulmonary embolism: Systematic review with meta-analysis. Vasc Med 2017;22:37-43.

25. Hirai T, Tate S, Dryer K, Jones D, Rosenberg J, Nathan S, et al. Electronic cardiac arrest triage score best predicts mortality after intervention in patients with massive and submassive pulmonary embolism. Catheter Cardiovasc Interv 2018. Doi: 10.1002/ ccd.27624.

26. Kolkailah AA, Hirji S, Piazza G, Ejiofor JI, Ramirez Del Val F, Lee J, et al. Surgical pulmonary embolectomy and catheter-directed thrombolysis for treatment of submassive pulmonary embolism. J Card Surg 2018;33:252-9.

27. Wang TF, Squizzato A, Dentali F, Ageno W. The role of thrombolytic therapy in pulmonary embolism. Blood 2015;125:2191-9. 